\title{
Dual Roles of CD38 in Autophagy
}

\author{
Xianwang Wang1,2*, Jiaxing Song3 ${ }^{3}$ Zijun Wu1 ${ }^{1}$, Buqun Fan1, Xiameng Mode1 \\ ${ }^{1}$ Laboratory of Neuronal Network and Brain Diseases Modulation, School of Medicine, Yangtze University, Jingzhou, China \\ ${ }^{2}$ The First Affiliated Hospital \& The First School of Clinical Medicine, Yangtze University, Jingzhou, China \\ ${ }^{3}$ Department of Pharmacology, School of Medicine, Shenzhen University, Shenzhen, China \\ Email: *xwshine@yangtzeu.edu.cn,87597782@qq.com,963512631@qq.com,1095083869@qq.com,1599596379@qq.com
}

How to cite this paper: Wang, X.W., Song J.X., Wu, Z.J., Fan, B.Q. and Mode X.M. (2017) Dual Roles of CD38 in Autophagy. Yangtze Medicine, 1, 8-19.

https://doi.org/10.4236/ym.2017.11002

Received: October 5, 2016

Accepted: March 27, 2017

Published: March 30, 2017

Copyright $\odot 2017$ by authors and Scientific Research Publishing Inc. This work is licensed under the Creative Commons Attribution International License (CC BY 4.0).

http://creativecommons.org/licenses/by/4.0/

\begin{abstract}
CD38 is a versatile, ubiquitously expressed protein that was identified as a multifunctional enzyme. Recently, cumulating evidence has suggested that CD38 is involved in autophagy, which is an evolutionarily conserved lysosomal degradation and recycling system. Acting as a enzyme, CD38 utilizes nicotinamide adenine dinucleotide phosphate (NADP) to synthesize nicotinic acid adenine dinucleotide phosphate (NAADP), which acts as a key messenger for $\mathrm{Ca}^{2+}$-mobilizing in lysosome by targeting two-pore channels (TPCs) or transient receptor potential mucolipins (TRPMLs). Multiple studies have indicated that CD38 is involved in autophagy by modulating intracellular $\mathrm{Ca}^{2+}$ signaling. However, the control of autophagy by CD38 signaling is the subject of two contrary views. The autophagosomes trafficking and fusion with lysosomes to form autolysosomes are crucial steps in autophagy. On the one hand, the available evidence indicates that lysosome trafficking and fusion to autophagosomes is positively modulated by CD38. On the other hand, overexpression of TPC2, which is positively modulated by CD38, was shown to promote the accumulation of autophagosomes, thus suppress autophagy. This review will reveal the interesting contrary dual roles of CD38 in autophagy, and critical insight into the molecular mechanisms of CD38 in autophagy regulation.
\end{abstract}

\section{Keywords}

CD38, Autophagy, Calcium, NAADP, Lysosome, Autophagosome

\section{CD38 and Calcium Signaling}

\subsection{The Function and Basic Structure of CD38}

CD38 is a type II membrane protein, originally identified as a cell surface differentiation marker in B lymphocytes, and later found to be expressed ubiquitously [1] [2]. In fact, CD38 is a versatile molecule with big ambitions. Although early studies revealed that CD38 serves as a differentiation antigen on cell sur- 
face, multiple studies have indicated a more diverse role for CD38 in many physiological and pathological processes, including $\mathrm{Ca}^{2+}$ signaling, autophagy, tumorigenesis, autism spectrum disorder (ASD), richter syndrome, acquired immune deficiency syndrome (AIDS) and type II diabetes [2]-[14].

As a transmembrane protein, human CD38 is a $46-\mathrm{kDa}$ glycoprotein, which consists of three conserved regions: a short $\mathrm{N}$-terminal topological domain (NTD, residues 1 - 21), a single transmembrane helix domain (TMD, residues 22 44) and a long C-terminal catalytic domain (CCD, residues 45 - 300) (Figure 1). Some key amino residues in $\mathrm{C}$-terminal catalytic region are responsible for nucleosidase activity of CD38. Mutations of the three residues W125F, W189G and E226G, affect the catalytic activity and subsequent calcium signaling [13]. CD38 catalyzed synthesis of cADPR, which increased intracellular $\mathrm{Ca}^{2+}$ and resulted in microglial activation and activation-induced cell death [14]. Moreover, CD38 contains some variations in single nucleotide that occur at specific sites in the genome, called single-nucleotide polymorphisms (SNPs), which have been used in genome-wide association studies (GWAS) as a key marker in gene mapping related to human diseases. The SNP position of CD38 at residues R140 (corresponding to rs1800561) is closely related to ASD or type II diabetes [9] [11]. Another SNP site rs6449182 at P184 is associated with richter syndrome [9] [10] [11]. Besides, the three disulfide bonds of CD38 are sponsored by cysteine residues between C160 and C173, C254 and C275, C278 and C296 [13]. The Nlinked glycosylation modifications are usually occurring at four asparagine residues N100, N164, N209 and N219 [15]. These disulfide bonds and glycosylation modifications might play important roles in the topology of CD38 [4].

\subsection{CD38, a Key Messager for Intracellular $\mathrm{Ca}^{2+-}$ Mobilizing}

Generally speaking, intracellular $\mathrm{Ca}^{2+}$ oscillation arise either from its release

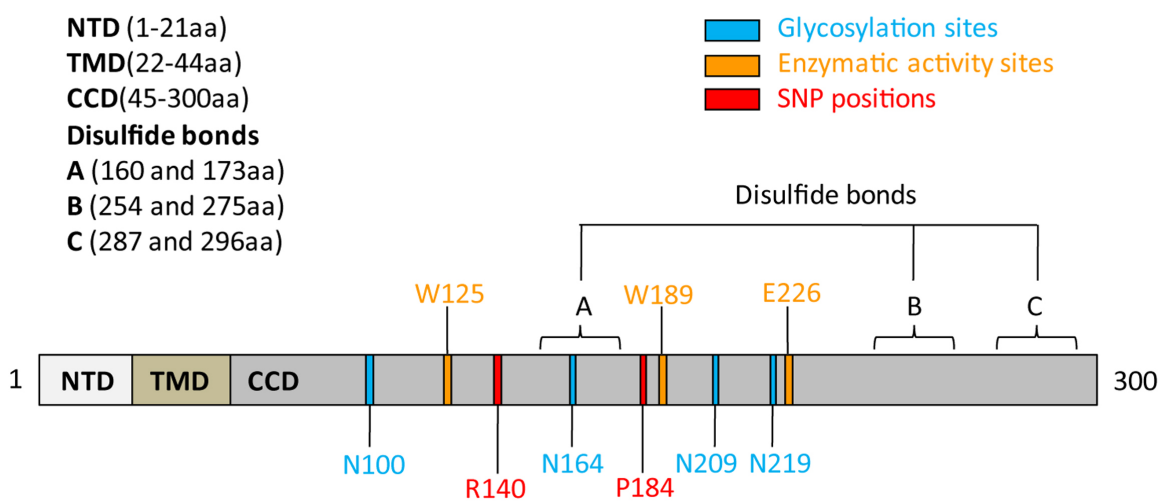

Figure 1. The structure of human CD38. Human CD38 contains three conserved domains: a short N-terminal topological domain (NTD, residues 1 - 21), a single transmembrane helix domain (TMD, residues 22 - 44) and a long C-terminal catalytic domain (CCD, residues 45 - 300). Three residues W125, W189 and E226 are responsible for nucleosidase activity of CD38. Two SNP positions occur at residues R140 and P184. The three disulfide bonds of CD38 are sponsored by cysteine residues between C160 and C173, C254 and C275, C278 and C296. The N-linked glycosylation modifications are occur at four asparagine residues N100, N164, N209 and N219. 
from intracellular stores, such as endoplasmic reticulum (ER), endolysosome and mitochondria, or by modulating $\mathrm{Ca}^{2+}$ channels at the plasma membrane, including voltage-dependent calcium channels (VDCCs), $\mathrm{Na}^{+} / \mathrm{Ca}^{2+}$ exchanger (NCX), plasma membrane calcium-transporting ATPases (PMCAs), cyclic nucleotide-gated ion channels (CNGCs), $\alpha$-amino-3-hydroxy-5-methyl-4-isoxazolepropionic acid receptor (AMPAR) and N-methyl-D-aspartate receptor (NMDAR) [2] [16].

As we known, Streb et al. discovered the $\mathrm{Ca}^{2+}$-mobilizing activity of inositol 1,4,5-triphosphate $\left(\mathrm{IP}_{3}\right)$, which targets $\mathrm{IP}_{3}$ receptor $\left(\mathrm{IP}_{3} \mathrm{R}\right)$ in ER and stimulates ER-Ca ${ }^{2+}$ release [2] [17]. In addition to $\mathrm{IP}_{3}$, cyclic ADP-ribose (cADPR) and nicotinic acid adenine dinucleotide phosphate (NAADP) are also identified as important intracellular $\mathrm{Ca}^{2+}$ messengers, which elicit intracellular $\mathrm{Ca}^{2+}$ flux from either ER or endolysosome. As a multifunctional enzyme, CD38 is responsible for the synthesis of CADPR and NAADP from NAD and NADP, respectively [2] [3]. Besides, CD38 also plays a critical role in the degradation of NAADP by hydrolyzing NAADP to ADP-ribose 2'-phosphate [18]. It is known that both $\mathrm{IP}_{3}$ and $C A D P R$ trigger $\mathrm{Ca}^{2+}$ release from the ER via the $\mathrm{IP}_{3} \mathrm{R}$ and ryanodine receptors (RYR), respectively. However, the other potent $\mathrm{Ca}^{2+}$-mobilizing messenger NAADP drive $\mathrm{Ca}^{2+}$ flux from the endolysosomal pools by targeting the two-pore channels (TPCs) or transient receptor potential mucolipin-1 (TRPML1) [3] [19] [20]. Intriguingly, the CD38/NAADP-mediated lysosome $\mathrm{Ca}^{2+}$ signaling have been shown to participate in the physiological regulation of cell functions or activities, such as autophagy and apoptosis [21]. Cumulating evidence has suggested that autophagosomes trafficking and fusion with lysosomes is a NAADP/ $\mathrm{Ca}^{2+}$ signaling dependent process [22]. Thus, modulating the production of NAADP in cells by CD38 may be play important roles in lysosomal functions, especially autolysosomes formation and autophagic contents elimination.

\section{Autophagy: A Brief Introduction}

Autophagy, also called self-eating, a natural and evolutionarily conserved lysosomal degradation and renovation process to hydrolyze unused or dysfunctional cellular components, such as long-lived or misfold proteins and useless organelles [23]. Actually, the basal autophagy activity is essential for cell homeostasis, involving cell growth, survival, development and death. The levels of autophagy must be precisely regulated, as indicated by the fact that dysfunctional autophagy has been related to wide ranges of human diseases, such as cancer [24] [25], neurodegenerative diseases [26], myopathies [27], gastrointestinal disorders [28], heart and liver diseases [29]. The most interesting is the study about autophagy and cancer. There are dual roles of autophagy in tumorigenesis [25] [30]. On the one hand, autophagy was thought to be a tumor-suppression mechanism. In normal cells and tissues, autophagy usually acts as a tumor suppressor by autophagy-mediated recycling [30] [31]. By preventing the toxic accumulation of damaged protein and organelles, particularly mitochondria, autophagy resists oxidative stress and oncogenic signaling, which inhibits malignancy. One the 
other hand, autophagy also plays tumor-promoting roles. In most contexts, cancers can induce autophagy to survive microenvironmental stress and to increase growth and aggressiveness [25] [30].

In recent years, autophagy has attracted great interest in the biological and medical sciences. Actually, there are at least three types or pathways of autophagy, including macroautophagy, microautophagy and chaperone-mediated autophagy [32]. One common hallmark of autophagy is mediated by the multiple autophagy-related (Atg) genes and their associated enzymes. The main and most common pathway, macroautophagy, usually called autophagy, is an intracellular recycling system, used primarily to mediate bulk degradation of superfluous or damaged cytosolic materials [23]. This autophagy processes include several steps: 1) induction and formation of a double membrane vesicle known as an autophagosome, 2) autophagosomes trafficking and fusion with lysosomes to form autolysosomes, and 3) degradation and recycling of autophagic contents via acidic lysosomal hydrolases (Figure 2).

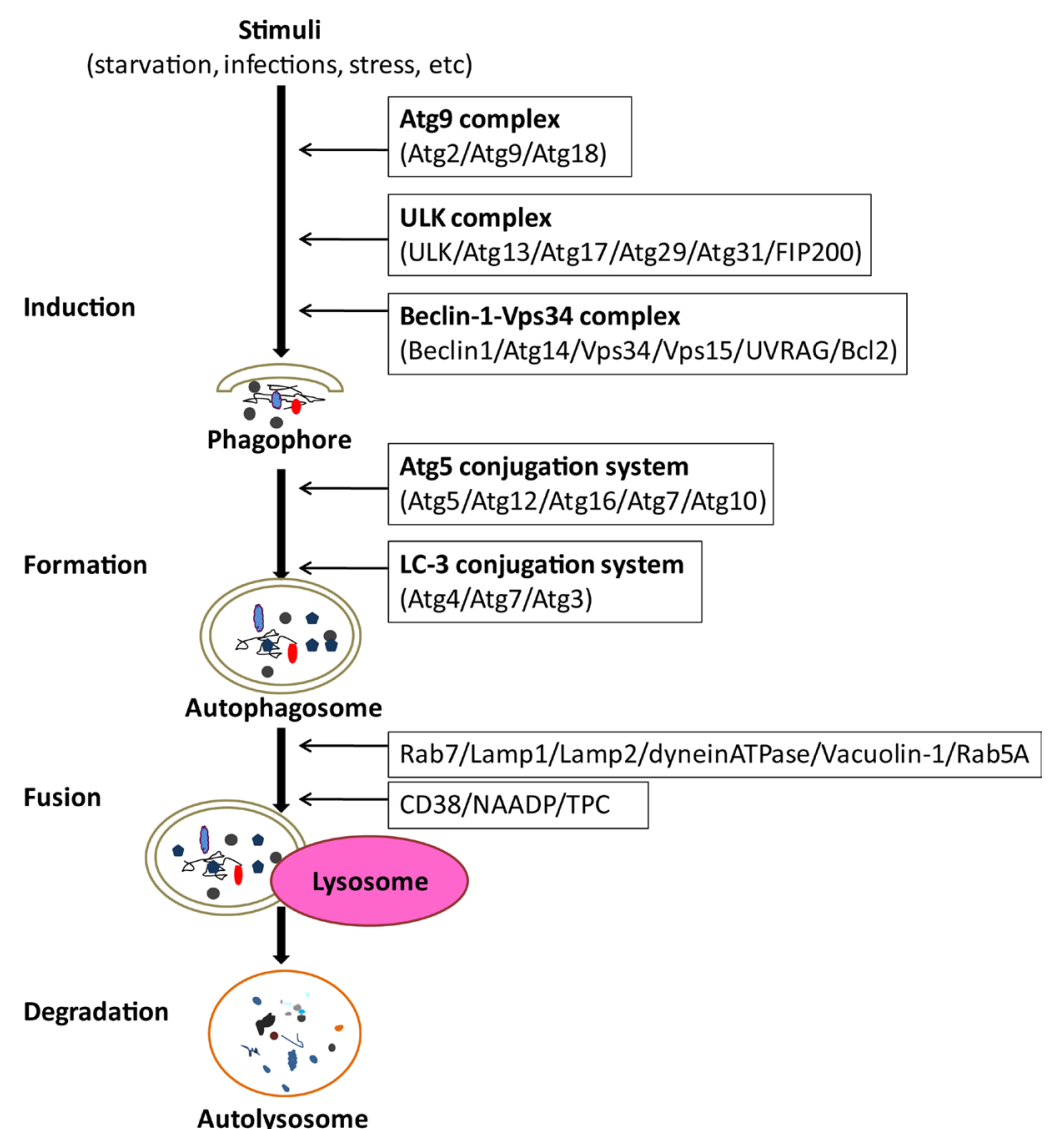

Figure 2. The overview of autophagy. The Atg9 complex, ULK complex and Beclin-1-Vps34 complex are important for induction of autophagosome. Besides, autophagosome formation also requires both LC3-II and Atg12-Atg5Atg16L conjugation systems. The autophagosomes trafficking and fusion with lysosomes to form autolysosomes is controlled by a lot of factors, including Rab7, Rab5A, Lamp1/Lamp2, dynein ATPase, Vacuolin-1, TRPMLs and TPCs. In the breakdown step, autophagic materials or contents are hydrolyzed by lysosomal enzymes in autolysosome. 
As an evolutionarily ancient response to cellular stress, cell autophagy can be evoked by a wide variety of stresses, including nutrition starvation, bacterial and viral infection, drought, salt, senescence and oxidative stress. Indeed, the autophagy process is executed by more than 30 Atg factors and associated proteins in response to stimuli. It is known that autophagy is initiated at the phagophore assembly site (PAS) in yeast [33]. Yamamoto et al. demonstrated that the Atg13mediated supramolecular assembly is responsible for autophagy initiation [34]. Yao et al. indicated that the Atg1 complex bond to Atg9-vesicles, thus facilitating the induction of autophagy [33]. In fact, autophagosome formation is the most complicated event, which is driven by series larger molecular complexes. As illustrated in Figure 2, autophagy induction is controlled by the Unc-51-Like kinase (ULK, also called Atg1) complexes [34] [35] [36], Atg9 complexes [33] and autophagosome formation requires Beclin-1-Vps34 complexes [23] [37]. Besides, the LC3-II (as ortholog of Atg8) and Atg12-Atg5-Atg16L conjugation systems are also essential for the autophagosomal formation [29] [35]. In addition to Atg proteins, many other factors and proteins, including Ras-related protein Rab7/5A (Rab7/5A), Lysosomal-associated membrane protein 1/2 (Lamp1/ Lamp2), ATPase, Vacuolin-1, TPCs and TRPMLs have been implicated in regulating autophagosomal lysosomal fusion process [35] [38] [39] [40].

In the end, the completion of autophagy depends on lysosomal activity, deficiencies in autophagosomal lysosomal fusion and degradation can lead to the accumulation of autophagosomes, ultimately impairing cells or resulting in cell death [41]. Accordingly, the mechanisms of the control for lysosome function are emerging as an important theme for autophagy-lysosomal cargos digestion.

\section{CD38 and Autophagy}

Because lysosomes play an important role in autophagy, the regulation mechanism of lysosomal functions is considered as the key element for autophagy. The fact that autophagy involves degradation by the autolysosome, which is formed by conjugation of an autophagasome and a lysosome, suggests that CD38/ NAADP signaling might be implicated in this process (Figure 3). It is known that CD38-mediated regulation of lysosome function contributes to autophagic flux or autophagy maturation. However, as listed in Table 1, there is paradox about the effect of $\mathrm{CD} 38$ on autophagy. The summary of these evidences are described as below.

\subsection{The Positive Regulation of CD38 in Autophagy}

Most lines of evidence support the positive regulation of CD38 in autophagy. Cumulating evidence has suggested that lysosome trafficking and fusion to autophagosomes is positively modulated by NAADP via CD38 enzymatic activity [19] [42]. Deficiency of CD38 resulted in a defective autophagic event in coronary arterial media of mice [22] [43]. Inhibition of CD38 by CD38 shRNA or nicotinamide leads to accumulation of p62 and autophagosomes, suppressing lysosome fusion to autophagosomes [44]. Moreover, in indolocarbazole induced 


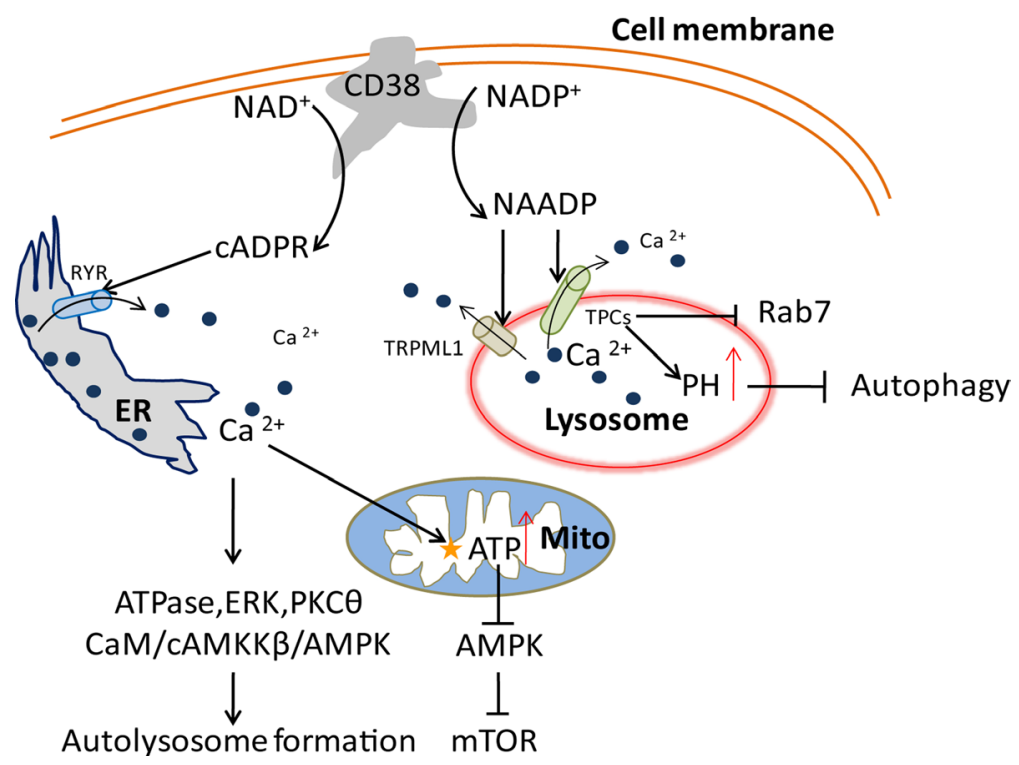

Figure 3. The potential mechanisms of CD38 in autophagy regulation. CD38 acts as a $\mathrm{NAD}^{+}$dependent enzyme, which catalyzes the synthesis of cADPR and NAADP from $\mathrm{NAD}^{+}$and NADP ${ }^{+}$, respectively. Then, the cytosolic cADPR and NAADP target to RYR on ER and TPC or TRP-ML1 on lysosome, modulating ER-Ca ${ }^{2+}$ signaling and lysosome- $\mathrm{Ca}^{2+}$ signaling, respectively. On the one hand, increasing of intracellular $\mathrm{Ca}^{2+}$ leads to activation of dynein ATPase, ERK, PKC $\theta$ or $\mathrm{CAMKK} \beta /$ AMPK signaling pathways, thus facilitates the formation of autolysosome. On the other hand, $\mathrm{Ca}^{2+}$ releases promote ATP synthesis in mitochondria (Mito) and AMPK inhibition, lead to suppression of mTOR and autophagy. Moreover, releasing of $\mathrm{Ca}^{2+}$ from lysosome by TPC2 or TRPML-1 has been suggested to inhibit Rab7 and alkalinizing lysosomal pH, thereby results in suppression of autophagosomal-lysosomal fusion, accumulation of autophagosomes in cytosol and subsequently autophagy impairment.

cell autophagy, CD38 expression and the LC3-II/LC3-I ratio are significantly elevated in response to ZW2-1 [45]. Subsequent studies showed that enzymatic activity of CD38 to produce NAADP plays an essential role in the following breakdown of the autophagic contents [19]. When nucleosidase activity of CD38 is disrupted or NAADP production is decreased, the formation of autolysosomes and degradation of autophagic vesicles also become impaired, which promotes cell dedifferentiation, proliferation and growth [19]. Besides, NAADP is also reported to increase acidic vesicular organelle formation and the expression levels of LC3II and Beclin-1 [46]. It is known that ROS promote autophagy maturation by regulating dynein-mediated autophagosomes trafficking [47]. High glucose induced ROS production results in the activation of dynein ATPase, which further triggers autophagosomes trafficking and fusion with lysosomes [47]. Moreover, antagonism of NAADP mediated $\mathrm{Ca}^{2+}$ signaling with NED-19 and pyridoxalphosphate-6-azophenyl-2',4'-disulfonic acid (PPADS) eliminated ROSevoked lysosomes $\mathrm{Ca}^{2+}$ flux and dynein activation [47].

\subsection{The Negative Regulation of CD38 in Autophagy}

In contrast, CD38 is also involved in inhibition of autophagy. Some lines of evi- 
Table 1. The roles of CD38 in autophagy. Abbreviation: Embryonic stem cells, ESC; Chronic lymphocytic leukemia, CLL; Immature hematopoietic progenitors, IHP; Coronary arterial myocytes, CAM; Mouse glomerular podocytes, MGP; Vascular endothelial cells, VEC; Vascular smooth muscle cells, VSMC.

\begin{tabular}{|c|c|c|c|}
\hline Cell types & Potential Mechanism & $\begin{array}{l}\text { Effect to } \\
\text { autophagy }\end{array}$ & $\begin{array}{c}\text { References } \\
\text { (first author, year) }\end{array}$ \\
\hline CAM & NAADP-Lysosome-Ca ${ }^{2+}$ & Positive & Zhang, 2010 \\
\hline CAM & Autophagic flux & Positive & $\mathrm{Xu}, 2011$ \\
\hline Astrocytes & NAADP/TPCs, LC3-II, Beclin-1 & Positive & Pereria, 2011 \\
\hline VEC, VSMC & NAADP/TRPML1/TPCs & Positive & Li, 2013 \\
\hline MGP & LC3-II & Positive & Xiong, 2013 \\
\hline CAM & Autophagic flux & Positive & Zhang, 2014 \\
\hline CAM & Dynein ATPase & Positive & $\mathrm{Xu}, 2014$ \\
\hline B cells & Blimp1, Beclin-1/p62 & Positive & Yuan, 2014 \\
\hline 293T cells & $\begin{array}{c}\text { NAADP/TRPML1, } \\
\text { LRRK2/CaMKK/AMPK }\end{array}$ & Negative & Gomez-Suaga, 2012 \\
\hline CLL cells & Beclin-1, Bcl-2, Autophagic flux & Negative & Bologna, 2013 \\
\hline Hela cells, ESC & $\begin{array}{c}\mathrm{NAADP} / \mathrm{TPC} 2 / \mathrm{Ca}^{2+} \text { signaling, } \\
\text { lysosomal } \mathrm{pH}\end{array}$ & Negative & Lu, 2013 \\
\hline ESC & NAADP/TPC2/ $\mathrm{Ca}^{2+}$ Signaling & Negative & Lu, 2013 \\
\hline IHP & Autophagic flux & Negative & Gomez-Puerto, 2016 \\
\hline
\end{tabular}

dence indicate that $\mathrm{CD} 38$ is a autophagy suppressor. Deficiency of CD150 is associated with increased expression levels of CD38 and inhibited phosphorylation of p38, JNK1/2, Bcl-2 and autophagic flux [7]. Moreover, CD38 deficiency immature hematopoietic progenitors show a higher autophagic flux as shown by analysis of LC3-II and p62 levels, as well as flow cytometry-based autophagic vesicle quantification [48]. NAADP as a potent $\mathrm{Ca}^{2+}$ mobilizing messenger, which targets the TRPMLs or TPCs and triggers $\mathrm{Ca}^{2+}$ release from the endolysosomal stores [19]. As we known, the members of the TRPML constitute a family of evolutionarily conserved cation channels that play crucial roles in endolysosomal vesicles [20] [49]. TRPMLs localize to endolysosomes and facilitate $\mathrm{Ca}^{2+}$-dependent fusion between autophagosomes and lysosomes resulting in lysosomal degradation of autophagic material [20]. It is known that TPCs have recently emerging as the targets for NAADP, which are crucial for appropriate basal and induced autophagic flux in cardiomyocytes [50]. For instance, overexpression of TPC2 in vitro suppressed autophagosomal-lysosomal fusion, thereby resulting in the decreasing levels of LC3-II and p62 and the accumulation of autophagosomes, suggesting that inhibition of autophagy progression by TPC2 [39] [41]. Thus, NAADP and its receptors (TRPMLs or TPCs) in lysosome may play crucial roles in lysosomal functions and subsequent autophagy events.

\subsection{The Potential Mechansim of CD38 in Autophagy}

It is well established that intracellular $\mathrm{Ca}^{2+}$ is one important regulators of autophagy [21]. Firstly, intracellular $\mathrm{Ca}^{2+}$-mobilization stimulates calmodulin (CaM), 
ERK and $\operatorname{PKC} \theta$ signaling, thereby mediating autophagy progression. Of note, overexpression of leucine-rich repeat kinase 2 (LRRK2) stimulates an increase in autophagy initiation through $\mathrm{Ca}^{2+}$-dependent activation of a CaMKK $\beta /$ adenosine monophosphate (AMP)-activated protein kinase (AMPK) pathway [51] [52]. Moreover, $\mathrm{Ca}^{2+}$ also inhibits autophagy by promoting mitochondria ATP, which inhibits CaMKK $\beta /$ AMPK pathway [21]. Thus, CD38 triggers intracellular $\mathrm{Ca}^{2+}$ flux from ER and lysosome can also result in opposite outcomes of autophagy directly, depending on multiple stimuli and the different cellular state. As illustrated in Figure 3, CD38 catalyzes the synthesis of cADPR and NAADP from NAD and NADP, respectively. These two messengers target to RYR and TPC or TRP-ML1, modulating ER-Ca ${ }^{2+}$ signaling and lysosome- $\mathrm{Ca}^{2+}$ signaling, respectively. On the one hand, increasing of intracellular $\mathrm{Ca}^{2+}$ leading to activation of dynein ATPase, ERK, PKC $\theta$ or $\mathrm{cAMKK} \beta /$ AMPK signaling pathways, thus facilitates the formation of autolysosome. On the other hand, $\mathrm{Ca}^{2+}$ releases promoting ATP synthesis in mitochondria and AMPK inhibition, leading to suppression of mTOR and autophagy. In addition, releasing of $\mathrm{Ca}^{2+}$ from lysosome by TPC 2 or TRPML-1 have been suggested to inhibit Rab7 and alkalinizing lysosomal $\mathrm{pH}$, thereby resulting suppression of autophagosomal-lysosomal fusion, accumulation of autophagosomes in cytosol and subsequently autophagy impairment.

Indeed, CD38 is involved in various stimuli induced autophagy. FasL has been shown to significantly increase NAADP production and intracellular $\mathrm{Ca}^{2+}$ flux in CD38 overexpressed mice [53]. In experimental autoimmune myocarditis mouse model, IL-17 promote B cell autophagy by controlling B-lymphocyte- induced maturation protein-1 (Blimp-1) expression and $\mathrm{CD} 38^{(+)} \mathrm{CD} 138^{(+)} \mathrm{B}$ cell percentages [54]. Not surprisingly, CD38 is possibly implicated in autophagy by variety of mechanisms. Lu et al. demonstrated that Vacuolin-1 suppress general endosomal-lysosomal degradation by decreasing $\mathrm{V}$-ATPase activity to enhance lysosomal $\mathrm{pH}$ in HeLa cells [38]. Of interest, in addition to CD38, other $\mathrm{NAD}^{+}$dependent enzymes, such as silent information regulator 2-related enzymes (Sirtuins) and the polymerase (ADP-ribose) polymerases (PARPs), act as the key mediators of macro autophagocytotic cell death [55] [56]. There is a potential crosstalk among CD38, Sirtuins and PARPs in autophagic cell death and survival pathways [56].

Taken together, there are several potential mechanisms of CD38 in autophagy, including 1) mobilizing intracellular $\mathrm{Ca}^{2+}$ release by producing cADPR and NAADP , 2) affecting the functions of lysosome by regulating the activity of lysosome related proteins, e.g. TPC2, Vacuolin-1, and 3) crosstalking with other $\mathrm{NAD}^{+}$-dependent enzymes, e.g. Sirtuins, PARPs. Thus, continuing to study the mechanism of $\mathrm{CD} 38$ and its related proteins in autophagy is very interesting works.

\section{Conclusion}

In conclusion, $\mathrm{CD} 38$ acts as an important autophagy modulator by predomi- 
nantly regulating $\mathrm{CD} 38 / \mathrm{NAADP} / \mathrm{Ca}^{2+}$ signaling mechanism. $\mathrm{CD} 38$ may be playing a dual effect on autophagy process in response to multiple different stimuli. A complete understanding the molecular mechanism of CD38 in autophagy remains in a fascinating challenge for future investigation.

\section{Acknowledgements}

This research is supported by Hubei Province Natural Science Foundation of China (grant No. 2016CFB180), Hubei Province Health and Family Planning Scientific Research Project (grant No. WJ2016Y07), Jingzhou Science and Technology Development Planning Project (grant no. JZKJ15063), the Open Fund of Laboratory of Neuronal Network and Brain Diseases Modulation in Yangtze University, the Development Fund from School of Medicine in Yangtze University, the Yangtze Fund for Youth Teams of Science and Technology Innovation and the College Students Innovative Entrepreneurial Training Program inYangtze University (grant No. 2016133). We thank Professor Hongwu Xin and his lab members for their valuable comments and advice.

\section{References}

[1] Jackson, D.G. and Bell, J.I. (1990) Isolation of a cDNA Encoding the Human CD38 (T10) Molecule, a Cell Surface Glycoprotein with an Unusual Discontinuous Pattern of Expression during Lymphocyte Differentiation. Journal of Immunology, $144,2811-2815$

[2] Zhao, Y., Graeff, R. and Lee, H.C. (2012) Roles of cADPR and NAADP in Pancreatic Cells. Acta Biochimica et Biophysica Sinica, 44, 719-729. https://doi.org/10.1093/abbs/gms044

[3] Galione, A. (2010) NAADP Receptors. Cold Spring Harbor Perspectives in Biology, 3, a004036.

[4] Zhao, Y.J., Zhu, W.J., Wang, X.W., Zhang, L.H. and Lee, H.C. (2015) Determinants of the Membrane Orientation of a Calcium Signaling Enzyme CD38. Biochimica et Biophysica Acta, 1853, 2095-2103. https://doi.org/10.1016/j.bbamcr.2014.10.028

[5] Li, T., et al. (2016) Immuno-Targeting the Multifunctional CD38 Using Nanobody. Scientific Reports, 6, Article No. 27055. https://doi.org/10.1038/srep27055

[6] Sasaki, M., Kakuda, Y., Miyakoshi, M., Sato, Y. and Nakanuma, Y. (2014) Infiltration of Inflammatory Cells Expressing Mitochondrial Proteins around Bile Ducts and in Biliary Epithelial Layer May Be Involved in the Pathogenesis in Primary Biliary Cirrhosis. Journal of Clinical Pathology, 67, 470-476.

https://doi.org/10.1136/jclinpath-2013-201917

[7] Bologna, C., et al. (2016) SLAMF1 Regulation of Chemotaxis and Autophagy Determines CLL Patient Response. The Journal of Clinical Investigation, 126, 181-194. https://doi.org/10.1172/JCI83013

[8] Chiarini, F., et al. (2012) A Combination of Temsirolimus, an Allosteric mTOR Inhibitor, with Clofarabine as a New Therapeutic Option for Patients with Acute Myeloid Leukemia. Oncotarget, 3, 1615-1628. https://doi.org/10.18632/oncotarget.762

[9] Munesue, T., et al. (2010) Two Genetic Variants of CD38 in Subjects with Autism Spectrum Disorder and Controls. Neuroscience Research, 67, 181-191. https://doi.org/10.1016/j.neures.2010.03.004 
[10] Aydin, S., et al. (2008) CD38 Gene Polymorphism and Chronic Lymphocytic Leukemia: A Role in Transformation to Richter Syndrome? Blood, 111, 5646-5653. https://doi.org/10.1182/blood-2008-01-129726

[11] Riebold, M., et al. (2011) All-Trans Retinoic Acid Upregulates Reduced CD38 Transcription in Lymphoblastoid Cell Lines from Autism Spectrum Disorder. Molecular Medicine, 17, 799-806.

[12] Antonelli, A., et al. (2002) Autoimmunity to CD38 and GAD in Type I and Type II Diabetes: CD38 and HLA Genotypes and Clinical Phenotypes. Diabetologia, 45, 1298-1306. https://doi.org/10.1007/s00125-002-0886-6

[13] Liu, Q., et al. (2005) Crystal Structure of Human CD38 Extracellular Domain. Structure, 13, 1331-1339. https://doi.org/10.1016/j.str.2005.05.012

[14] Mayo, L., et al. (2008) Dual Role of CD38 in Microglial Activation and Activation-Induced Cell Death. The Journal of Immunology, 181, 92-103. https://doi.org/10.4049/jimmunol.181.1.92

[15] Chen, R., et al. (2009) Glycoproteomics Analysis of Human Liver Tissue by Combination of Multiple Enzyme Digestion and Hydrazide Chemistry. Journal of Proteome Research, 8, 651-661. https://doi.org/10.1021/pr8008012

[16] Glazner, G.W., Chan, S.L., Lu, C. and Mattson, M.P. (2000) Caspase-Mediated Degradation of AMPA Receptor Subunits: A Mechanism for Preventing Excitotoxic Necrosis and Ensuring Apoptosis. The Journal of Neuroscience: The Official Journal of the Society for Neuroscience, 20, 3641-3649.

[17] Streb, H., Irvine, R.F., Berridge, M.J. and Schulz, I. (1983) Release of $\mathrm{Ca}^{2+}$ from a Nonmitochondrial Intracellular Store in Pancreatic Acinar Cells by Inositol-1,4,5-trisphosphate. Nature, 306, 67-69. https://doi.org/10.1038/306067a0

[18] Schmid, F., Bruhn, S., Weber, K., Mittrucker, H.W. and Guse, A.H. (2011) CD38: A NAADP Degrading Enzyme. FEBS Letters, 585, 3544-3548.

https://doi.org/10.1016/j.febslet.2011.10.017

[19] Li, P.L., Zhang, Y., Abais, J.M., Ritter, J.K. and Zhang, F. (2013) Cyclic ADP-Ribose and NAADP in Vascular Regulation and Diseases. Messenger, 2, 63-85. https://doi.org/10.1166/msr.2013.1022

[20] Venkatachalam, K., Wong, C.O. and Zhu, M.X. (2015) The Role of TRPMLs in Endolysosomal Trafficking and Function. Cell Calcium, 58, 48-56.

https://doi.org/10.1016/j.ceca.2014.10.008

[21] Decuypere, J.P., Bultynck, G. and Parys, J.B. (2011) A Dual Role for $\mathrm{Ca}(2+)$ in Autophagy Regulation. Cell Calcium, 50, 242-250. https://doi.org/10.1016/j.ceca.2011.04.001

[22] Zhang, Y., et al. (2014) Defective Autophagosome Trafficking Contributes to Impaired Autophagic Flux in Coronary Arterial Myocytes Lacking CD38 Gene. Cardiovascular Research, 102, 68-78. https://doi.org/10.1093/cvr/cvu011

[23] Kobayashi, S. (2015) Choose Delicately and Reuse Adequately: The Newly Revealed Process of Autophagy. Biological and Pharmaceutical Bulletin, 38, 1098-1103. https://doi.org/10.1248/bpb.b15-00096

[24] Brisson, L., et al. (2016) Lactate Dehydrogenase B Controls Lysosome Activity and Autophagy in Cancer. Cancer Cell, 30, 418-431. https://doi.org/10.1016/j.ccell.2016.08.005

[25] Mowers, E.E., Sharifi, M.N. and Macleod, K.F. (2016) Autophagy in Cancer Metastasis. Oncogene, 1-12. https://doi.org/10.1038/onc.2016.333

[26] Caccamo, A., Ferreira, E., Branca, C. and Oddo, S. (2016) p62 Improves AD-Like Pathology by Increasing Autophagy. Molecular Psychiatry, 1-9. 
[27] Lai, C.H., et al. (2016) Multi-Strain Probiotics Inhibit Cardiac Myopathies and Autophagy to Prevent Heart Injury in High-Fat Diet-Fed Rats. International Journal of Medical Sciences, 13, 277-285. https://doi.org/10.7150/ijms.14769

[28] Hernandez, C., et al. (2016) Aspirin-Induced Gastrointestinal Damage Is Associated with an Inhibition of Epithelial Cell Autophagy. Journal of Gastroenterology, 51, 691-701. https://doi.org/10.1007/s00535-015-1137-1

[29] Hofius, D., Munch, D., Bressendorff, S., Mundy, J. and Petersen, M. (2011) Role of Autophagy in Disease Resistance and Hypersensitive Response-Associated Cell Death. Cell Death and Differentiation, 18, 1257-1262. https://doi.org/10.1038/cdd.2011.43

[30] Amaravadi, R., Kimmelman, A.C. and White, E. (2016) Recent Insights into the Function of Autophagy in Cancer. Genes and Development, 30, 1913-1930. https://doi.org/10.1101/gad.287524.116

[31] White, E. (2015) The Role for Autophagy in Cancer. Journal of Clinical Investigation, 125, 42-46. https://doi.org/10.1172/JCI73941

[32] Sato, M., et al. (2016) Fluorescent-Based Evaluation of Chaperone-Mediated Autophagy and Microautophagy Activities in Cultured Cells. Genes to Cells. Devoted to Molecular and Cellular Mechanisms, 21, 861-873. https://doi.org/10.1111/gtc.12390

[33] Rao, Y., Perna, M.G., Hofmann, B., Beier, V. and Wollert, T. (2016) The Atg1-Kinase Complex Tethers Atg9-Vesicles to Initiate Autophagy. Nature Communications, 7, Article No. 10338. https://doi.org/10.1038/ncomms10338

[34] Yamamoto, H., et al. (2016) The Intrinsically Disordered Protein Atg13 Mediates Supramolecular Assembly of Autophagy Initiation Complexes. Developmental Cell, 38, 86-99. https://doi.org/10.1016/j.devcel.2016.06.015

[35] Karanasios, E., et al. (2016) Autophagy Initiation by ULK Complex Assembly on ER Tubulovesicular Regions Marked by ATG9 Vesicles. Nature Communications, 7, 12420. https://doi.org/10.1038/ncomms 12420

[36] Jung, C.H., et al. (2009) ULK-Atg13-FIP200 Complexes Mediate mTOR Signaling to the Autophagy Machinery. Molecular Biology of the Cell, 20, 1992-2003. https://doi.org/10.1091/mbc.E08-12-1249

[37] Funderburk, S.F., Wang, Q.J. and Yue, Z. (2010) The Beclin 1-VPS34 Complex-At the Crossroads of Autophagy and Beyond. Trends in Cell Biology, 20, 355-362. https://doi.org/10.1016/j.tcb.2010.03.002

[38] Lu, Y., et al. (2014) Vacuolin-1 Potently and Reversibly Inhibits AutophagosomeLysosome Fusion by Activating RAB5A. Autophagy, 10, 1895-1905. https://doi.org/10.4161/auto.32200

[39] Lu, Y., et al. (2013) Two Pore Channel 2 (TPC2) Inhibits Autophagosomal-Lysosomal Fusion by Alkalinizing Lysosomal pH. The Journal of Biological Chemistry, 288, 24247-24263. https://doi.org/10.1074/jbc.M113.484253

[40] Gao, Y., et al. (2016) Golgi-Associated LC3 Lipidation Requires V-ATPase in Noncanonical Autophagy. Cell Death and Disease, 7, e2330. https://doi.org/10.1038/cddis.2016.236

[41] Lu, Y., Hao, B., Graeff, R. and Yue, J. (2013) NAADP/TPC2/Ca(2+) Signaling Inhibits Autophagy. Communicative and Integrative Biology, 6, e27595. https://doi.org/10.4161/cib.27595

[42] Parrington, J. and Tunn, R. (2014) Ca(2+) Signals, NAADP and Two-Pore Channels: Role in Cellular Differentiation. Acta Physiologica, 211, 285-296. https://doi.org/10.1111/apha.12298

[43] Xu, M., et al. (2011) Lysosomal Regulation of Autophagy Efflux via CD38-Mediated 
Signaling in Mouse Coronary Arterial Myocytes. Hypertension, 58, E178-E178.

[44] Xiong, J., et al. (2013) Autophagy Maturation Associated with CD38-Mediated Regulation of Lysosome Function in Mouse Glomerular Podocytes. Journal of Cellular and Molecular Medicine, 17, 1598-1607. https://doi.org/10.1111/jcmm.12173

[45] Wang, W., Lv, M., Zhao, X. and Zhang, J. (2015) Developing a Novel Indolocarbazole as Histone Deacetylases Inhibitor against Leukemia Cell Lines. Journal of Analytical Methods in Chemistry, 2015, Article ID: 675053. https://doi.org/10.1155/2015/675053

[46] Pereira, G.J., et al. (2011) Nicotinic Acid Adenine Dinucleotide Phosphate (NAADP) Regulates Autophagy in Cultured Astrocytes. The Journal of Biological Chemistry, 286, 27875-27881. https://doi.org/10.1074/jbc.C110.216580

[47] Xu, M., et al. (2014) Enhancement of Dynein-Mediated Autophagosome Trafficking and Autophagy Maturation by ROS in Mouse Coronary Arterial Myocytes. Journal of Cellular and Molecular Medicine, 18, 2165-2175. https://doi.org/10.1111/jcmm.12326

[48] Gomez-Puerto, M.C., et al. (2016) Autophagy Proteins ATG5 and ATG7 Are Essential for the Maintenance of Human CD34(+) Hematopoietic Stem-Progenitor Cells. Stem Cells, 34, 1651-1663. https://doi.org/10.1002/stem.2347

[49] Zeevi, D.A., Lev, S., Frumkin, A., Minke, B. and Bach, G. (2010) Heteromultimeric TRPML Channel Assemblies Play a Crucial Role in the Regulation of Cell Viability Models and Starvation-Induced Autophagy. Journal of Cell Science, 123, 3112-3124. https://doi.org/10.1242/jcs.067330

[50] Garcia-Rua, V., et al. (2016) Endolysosomal Two-Pore Channels Regulate Autophagy in Cardiomyocytes. The Journal of Physiology, 594, 3061-3077. https://doi.org/10.1113/JP271332

[51] Gomez-Suaga, P., et al. (2012) Leucine-Rich Repeat Kinase 2 Regulates Autophagy through a Calcium-Dependent Pathway Involving NAADP. Human Molecular Genetics, 21, 511-525. https://doi.org/10.1093/hmg/ddr481

[52] Gomez-Suaga, P. and Hilfiker, S. (2012) LRRK2 as a Modulator of Lysosomal Calcium Homeostasis with Downstream Effects on Autophagy. Autophagy, 8, 692-693. https://doi.org/10.4161/auto.19305

[53] Zhang, F., Xia, M. and Li, P.L. (2010) Lysosome-Dependent Ca(2+) Release Response to Fas Activation in Coronary Arterial Myocytes through NAADP: Evidence from CD38 Gene Knockouts. American Journal of Physiology: Cell Physiology, 298, C1209-C1216. https://doi.org/10.1152/ajpcell.00533.2009

[54] Yuan, J., et al. (2014) Autophagy Contributes to IL-17-Induced Plasma Cell Differentiation in Experimental Autoimmune Myocarditis. International Immunopharmacology, 18, 98-105. https://doi.org/10.1016/j.intimp.2013.11.008

[55] Ma, Y.N.H., Chen, H., Li, J., Hong, Y., Wang, B., Wang, C., Zhang, J., Cao, W., Zhang, M., Xu, Y., Ding, X., Yin, S.K., Qu, X. and Ying, W. (2015) NAD ${ }^{+} / \mathrm{NADH}$ Metabolism and $\mathrm{NAD}^{+}$-Dependent Enzymes in Cell Death and Ischemic Brain Injury: Current Advances and Therapeutic Implications. Current Medicinal Chemistry, 22, 1239-1247. https://doi.org/10.2174/0929867322666150209154420

[56] Hassa, P.O. (2009) The Molecular "Jekyll and Hyde" Duality of PARP1 in Cell Death and Cell Survival. Frontiers in Bioscience, 14, U72-U73.

https://doi.org/10.2741/3232 
Submit or recommend next manuscript to SCIRP and we will provide best service for you:

Accepting pre-submission inquiries through Email, Facebook, LinkedIn, Twitter, etc. A wide selection of journals (inclusive of 9 subjects, more than 200 journals)

Providing 24-hour high-quality service

User-friendly online submission system

Fair and swift peer-review system

Efficient typesetting and proofreading procedure

Display of the result of downloads and visits, as well as the number of cited articles Maximum dissemination of your research work

Submit your manuscript at: http://papersubmission.scirp.org/

Or contact ym@scirp.org 